\title{
Evaluation of children with neurobrucellosis
}

\author{
Akbayram $\mathrm{S}^{1}$, Dogan $\mathrm{M}^{1}$, Peker $\mathrm{E}^{1}$, Bektas $\mathrm{MS}^{1}$, Caksen $\mathrm{H}^{1}$, Karahocagil $\mathrm{M}^{2}$ \\ Department of Pediatrics, School of Medicine, Yuzuncu Yil University, Van, Turkey. \\ doganmurat.md@gmail.com
}

\begin{abstract}
Background: Brucellosis is an endemic disease in many areas throughout the world. Central nervous system involvement is a serious complication of brucellosis with a ratio of $4-11 \%$ of all patients.

Aim: to describe our experience in diagnosis, treatment, and outcome of 25 pediatric patients with neurobrucellosis. Patients and methods: This study included a review of medical records of patients who were diagnosed with neurobrucellosis between March 2001 and March 2009. Patients who had both clinical findings consistent with neurobrucellosis and positive microbiologic/serologic examinations of CSF with abnormal CSF findings were enrolled in the study.

Results: The study included 25 patients between 1 and 15 years of age (mean 8.8 years), while 15 were males and 10 were females. Most of the patients (52\%) were in the age group of 5-9 years with male predominance. The distribution of cases showed density in June and February. The most commonly presented complaints were headache, fever and sweating while the most commonly observed findings were fever and meningeal irritation signs. All patients had positive cerebrospinal fluid agglutination test for brucellosis. Four different regimens were used based on ceftriaxone, doxycycline, cotrimoxasole, streptomycin, and rifampicin. One patient died, three patients were discharged with sequel, and the remaining patients (84 \%) were discharged with full recovery. Conclusion: Clinicians, especially those providing health services in endemic areas like Turkey, should keep in mind that neurobrucellosis can be involved in patients with unexplained symptoms like memory impairment or in patients diagnosed with meningitis (Tab. 5, Fig, 2, Ref. 39). Full Text in PDF www.elis.sk.

Key words: neurobrucellosis, treatment, diagnosis, children.
\end{abstract}

Brucellosis is the most common zoonotic infection in the world and annually more than 500,000 new cases occur (1). It is an endemic disease in many areas throughout the world including Mediterranean Basin, South and Central America, Eastern Europe, Asia, Africa, the Caribbean, and the Middle East (2). Turkey is one of those countries located on the shores of the Mediterranean Sea. In 2004, the incidence of brucellosis in Turkey was 25.67/100,000 population (3).

Humans can be infected by direct inoculation of microorganism through cuts and skin abrasions, by inhalation of infected aerosols, via conjunctiva, or ingestion of contaminated food such as unpasteurized milk, cheese made from unpasteurized milk, or raw meat (4). The central nervous system (CNS) involvement is a serious of brucellosis complication with a ratio of $4-11 \%$ of all patients while the clinical presentation is quite heterogeneous (5-8). The clinical presentation of neurobrucellosis includes peripheral neuropathy/radiculopathy, meningovascular involvement, meningoencephalitis, parenchymatous dysfunction and various degrees of behavioral abnormalities (9). Most commonly, a typi-

${ }^{1}$ Department of Pediatrics, School of Medicine, Yuzuncu Yil University, Van, Turkey, and ${ }^{2}$ Department of Infectious Diseases and Clinical Microbiology, School of Medicine, Yuzuncu Yil University, Van, Turkey

Address for correspondence: M. Dogan, MD, Department of Pediatrics, School of Medicine, Yuzuncu Yil University, Hafiziye Mah. K. Karabekir Cad. Arastirma Hastanesi Arkasi, Ugur Sitesi D blok 4. kat No: 9, 65100 Van, Turkey.

Phone: +90.506.5871817, Fax: +90.432 .2155281$ cal meningitis presents either as an only sign of infection or in context of a systemic disease (10-12).

In this study, we describe our experience in diagnosis, treatment, and outcome of 25 pediatric patients with neurobrucellosis. To the best of our knowledge, our series is the largest study on pediatric neurobrucellosis in literature.

\section{Patients and methods}

This is a retrospective study, which includes a review of medical records of patients who were admitted to Yuzuncu Yil University Medical School Hospital - provider of healthcare to patients from five different Eastern Turkey cities - and diagnosed with neurobrucellosis between March 2001 and March 2009.

\section{Diagnosis of neurobrucellosis}

Patients younger than 16 years, of either sex were included in the study. In addition, patients who had both clinical findings consistent with neurobrucellosis and positive microbiologic/serologic examinations of CSF with abnormal CSF findings were enrolled in the study. The diagnosis of neurobrucellosis requires to fulfill specific criteria as follows: (a) clinical features of illness compatible with a known neurobrucellosis syndrome, (b) typical CSF changes (presence of $>10 / \mathrm{mm}^{3}$, protein levels $>45 \mathrm{mg} / \mathrm{dL}$ or glucose levels $<40 \mathrm{mg} / \mathrm{dL}$ or less than $40 \%$ of the concomitant blood glucose level), (c) positive results of either blood or bone marrow or CSF culture or appropriate serological tests (e.g., agglu- 

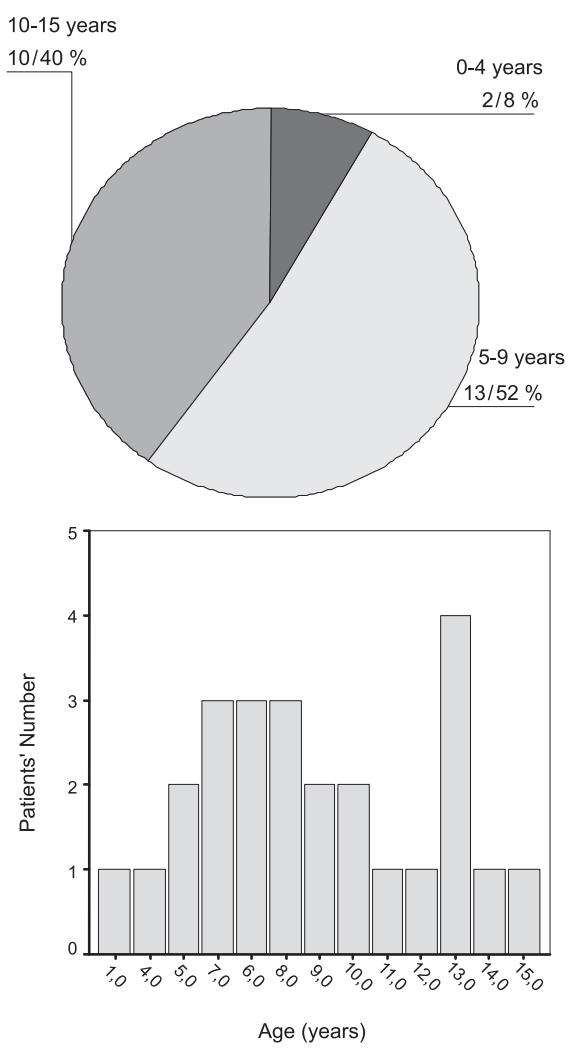

Fig. 1. The distribution of patients according to age group and age.

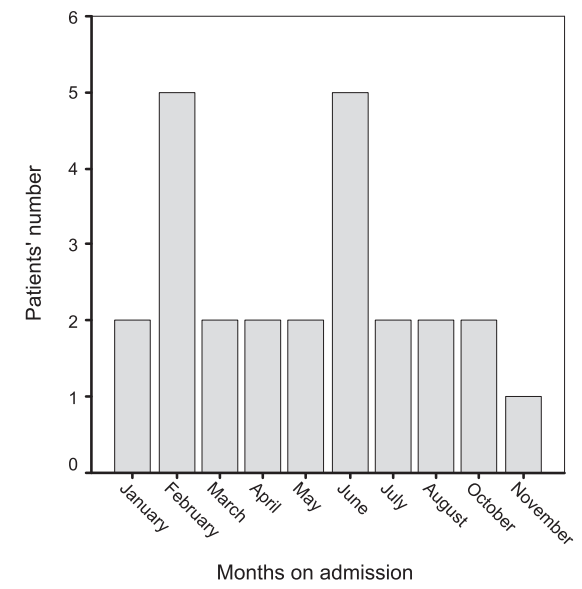

Fig. 2. The distribution of all patients according to month on admission.

tination test titers of $>1: 160$ in blood or any positive titer in CSF), (d) clinical improvement after starting the appropriate treatment, and (e) inability to prove a more suitable alternative diagnosis (13).

\section{Investigated parameters}

All patients' medical records were investigated in term of patients' age, gender, clinical symptoms and their duration at the time of admission, history of ingestion of unpasteurized milk or milk products, physical examination findings, family history for brucellosis, living area, occupation of patients or that of their
Tab. 1. Shows the distribution of patients according to complaints on admission.

\begin{tabular}{lcc}
\hline Complaints on admission & \multicolumn{2}{c}{$\begin{array}{c}\text { Frequency and percentage of } \\
\text { complaints on admission }\end{array}$} \\
\cline { 2 - 3 } & $\mathrm{N}$ & $\%$ \\
\hline Headache & 25 & 100 \\
Fever & 25 & 100 \\
Sweating & 25 & 100 \\
Weight loss & 12 & 48 \\
Back pain & 9 & 36 \\
Walking diffuculty & 2 & 8 \\
Neusea and vomiting & 25 & 100 \\
Weakness & 21 & 84 \\
Sensory loss in the extremities & 2 & 8 \\
Urinary incontinence & 2 & 8 \\
Fecal incontinence & 2 & 8 \\
Blurred vision & 4 & 16 \\
Double vision & 6 & 24 \\
Memory deficit & 2 & 8 \\
\hline
\end{tabular}

parents, use of antibiotics for brucellosis before admission, season and month on admission, laboratory results, complications, treatments, radiological examinations and outcome.

\section{Statistical analyses}

Statistical analyses were done by using SPSS 17.0 software package program. Nominal data were analyzed with Chi-square tests. Results were expressed as mean \pm SD when applicable. $\mathrm{P}<0.05$ was considered significant.

\section{Results}

The study included 25 patients between 1 and 15 years of age (mean 8.8 years), 15 (60\%) were males and 10 (40\%) were females. A statistically significant difference was found for gender $(\mathrm{p}<0.001)$. Most of the patients $(52 \%)$ were in an age group of 5-9 years (Fig. 1). The distribution of cases showed density in June (20\%) and February (20\%) (Fig. 2).

All patients with neurobrucellosis had an epidemiological risk factor for brucellosis. All were from the Eastern Turkey region of the city Van. Of 25 patients, 2 ( $8 \%$ ) were from Van city center, $13(52 \%)$ from town centers and 10 (40\%) were

Tab. 2. Shows the distribution of patients according to physical examination findings on admission.

\begin{tabular}{lcc}
\hline Physical examination findings & \multicolumn{2}{c}{ Frequency and percentage } \\
\cline { 2 - 3 } & $\mathrm{N}$ & $\%$ \\
\hline Fever & 25 & 100 \\
Meningeal irritation & 25 & 100 \\
Confusion & 19 & 76 \\
Hepatomegaly & 4 & 16 \\
Splenomegaly & 5 & 20 \\
Hypoesthesia & 1 & 4 \\
Convulsions & 12 & 48 \\
Diplopia & 6 & 24 \\
Pupil edema & 2 & 8 \\
Coma & 5 & 20 \\
Ataxia & 1 & 4 \\
\hline
\end{tabular}


Tab. 3. Shows hemogram, erythrocyte sedimentation rates, C-reactive protein levels and coagulation parameters of all patients on admission.

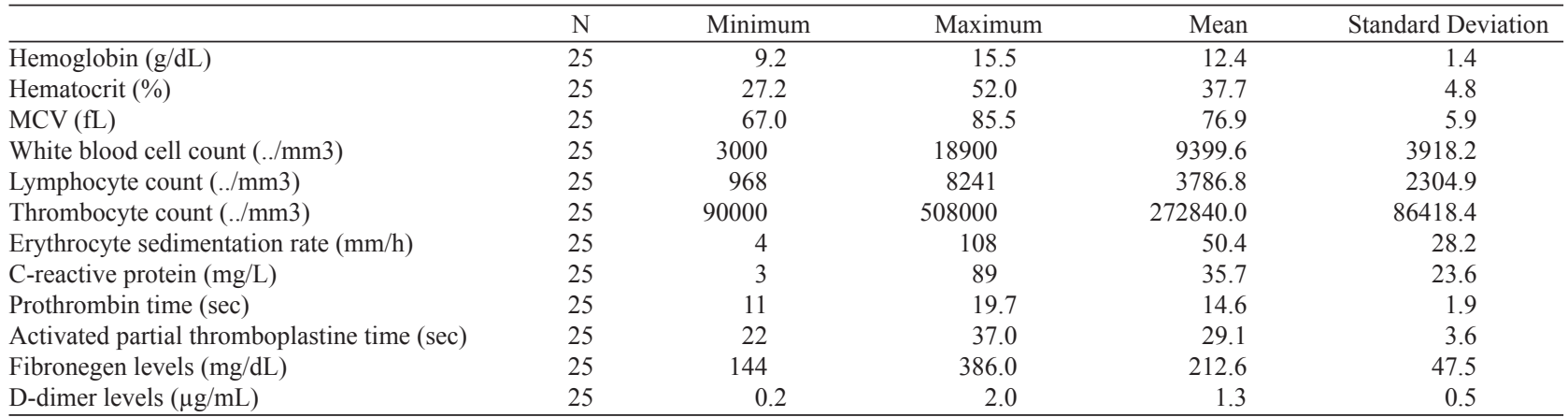

D-dimer levels $(\mu \mathrm{g} / \mathrm{mL})$

MCV: mean corpusculer volume, sec: second

Tab. 4. Shows the liver, renal function tests and serum electrolytes of all patients.

\begin{tabular}{|c|c|c|c|c|c|}
\hline & $\mathrm{N}$ & Minimum & Maximum & Mean & Standard Deviation \\
\hline Aspartate aminotransferase (U/L) & 25 & 12 & 346 & 56.8 & 65.5 \\
\hline Alanine amino transferase (U/L) & 25 & 9 & 480 & 57.0 & 93.4 \\
\hline Lactate dehydrogenase (U/L) & 25 & 214 & 1159 & 606.4 & 265.9 \\
\hline Blood urea nitrogen $(\mathrm{mg} / \mathrm{dL})$ & 25 & 5.5 & 22 & 11.6 & 4.6 \\
\hline Creatinine $(\mathrm{mg} / \mathrm{dL})$ & 25 & 0.3 & 0.7 & 0.5 & 0.1 \\
\hline Total bilirubin (mg/dL) & 25 & 0.2 & 0.8 & 0.4 & 0.2 \\
\hline Direct bilirubin (mg/dL) & 25 & 0.01 & 0.7 & 0.4 & 1.4 \\
\hline Alkaline phosphatase (U/L) & 25 & 103 & 549 & 231.1 & 104.4 \\
\hline Serum sodium $(\mathrm{mEq} / \mathrm{L})$ & 25 & 118 & 144 & 132.8 & 6.5 \\
\hline Serum potassium $(\mathrm{mEq} / \mathrm{L})$ & 25 & 3.3 & 5.6 & 4.5 & 0.5 \\
\hline Serum clorur $(\mathrm{mEq} / \mathrm{L})$ & 25 & 85 & 104 & 98.4 & 4.9 \\
\hline Serum glucose (mg/dL) & 25 & 63 & 139 & 97.0 & 19.4 \\
\hline Serum calcium (mg/dL) & 25 & 7.5 & 10.7 & 9.1 & 0.9 \\
\hline Serum phosphor (mg/dL) & 25 & 2.6 & 5.5 & 4.0 & 0.7 \\
\hline Serum albumin $(\mathrm{g} / \mathrm{L})$ & 25 & 2.7 & 4.7 & 3.5 & 0.5 \\
\hline Serum uric acid (mg/dL) & 25 & 2.0 & 12.0 & 5.6 & 2.8 \\
\hline
\end{tabular}

from villages. Twenty-three ( $92 \%$ ) of all patients were living in rural areas. Fifteen $(60 \%)$ had been consuming unpasteurized dairy products, $14(56 \%)$ had a history of brucellosis in their family members and 17 (68\%) patients had family members who were either farmers or herdsmen. No underlying disease was found in the patients.

The distribution of patients according to their complaints on admission is shown in Table 1. The most common presenting complaints were headache (100\%), fever (100\%), sweating (100\%), nausea and vomiting (100\%), weakness ( $84 \%)$, visual disorders (56\%), weight loss (48\%) and back pain (36\%). The mean duration of period between the appearance of symptoms and admission to hospital was $18 \pm 11.7$ days with a range of 3 to 60 days.

The findings on physical examinations are shown in Table 2. On physical examination, fever and meningeal irritation signs were observed in all patients. Additional confusion was noted in 19 (76 $\%$ ), convulsion in $12(48 \%)$, and papilledema in $2(8 \%)$ patients.

The laboratory findings of patients are shown in Tabs 3-5. Anemia was diagnosed in 8 (32\%), leukocytosis in $3(12 \%)$, leukopenia in $3(12 \%)$ and thrombocytopenia in one (4\%) patient. Additional increase in erythrocyte sedimentation rate, C-reactive protein and prothrombin time were found in 20 (80\%), 22 (88 $\%)$ and $10(40 \%)$ patients, respectively. Although the activated partial thromboplastine time and fibrinogen levels were found to be normal in all patients, D-dimer level was found to be increased in $23(92 \%)$ patients.

Serum electrolytes, serum bilirubin and renal function tests were normal in all patients. Six (24\%) patients had abnormal liver function tests.

The findings of cerebrospinal fluid examination are shown in Table 5. All patients had positive cerebrospinal fluid agglutination tests for brucellosis. Cerebrospinal and hematological cultures were positive for Brucella melitensis in five patients.

On cranial tomography and magnetic resonance imaging examinations, concomitant brain abscess and hydrocephalus were diagnosed only in one patient. In the remaining patients, the latter examinations yielded normal findings. The patient with brain abscess had also an abnormal liver function test. Another patient had hydrocephalus with infarction in left thalamic regions on magnetic resonance imaging. The patient had a ventriculoperitoneal shunt inserted because of hydrocephalus. After being treated with ceftriaxone plus rifampicin plus cotrimaxosole, the patient was discharged with sequel.

Unilateral abducens palsy was found in six (24\%) patients. After being treated, all of these patients were discharged without sequel. 
Tab. 5. Shows the cerebrospinal fluid examinations of all patients.

\begin{tabular}{|c|c|c|c|c|c|c|c|c|c|}
\hline 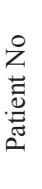 & 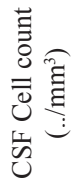 & 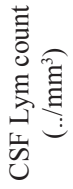 & 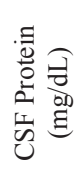 & 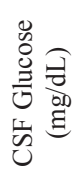 & 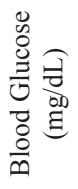 & 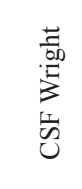 & 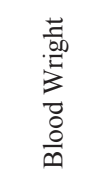 & 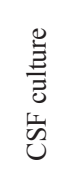 & 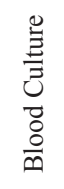 \\
\hline 1 & 0 & 0 & 108 & 89 & 117 & $1 / 40$ & $1 / 1280$ & - & - \\
\hline 2 & 340 & 100 & 146 & 49 & 63 & $1 / 80$ & $1 / 640$ & - & - \\
\hline 4 & 180 & 50 & 134 & 26 & 93 & $1 / 80$ & $1 / 320$ & - & - \\
\hline 5 & 500 & 350 & 61,1 & 41 & 98 & $1 / 160$ & $1 / 640$ & - & - \\
\hline 6 & 80 & 60 & 27,4 & 63 & 139 & $1 / 40$ & $1 / 320$ & - & - \\
\hline 7 & 150 & 80 & 82 & 45 & 88 & $1 / 80$ & $1 / 160$ & - & - \\
\hline 8 & 230 & 120 & 129 & 22 & 96 & $1 / 320$ & $1 / 1280$ & B.m. & B.m. \\
\hline 9 & 160 & 90 & 118 & 31 & 80 & $1 / 160$ & $1 / 1280$ & - & - \\
\hline 10 & 120 & 70 & 89 & 41 & 128 & $1 / 40$ & $1 / 160$ & - & - \\
\hline 11 & 170 & 90 & 98 & 31 & 74 & $1 / 40$ & $1 / 160$ & - & - \\
\hline 13 & 410 & 200 & 147 & 45 & 100 & $1 / 160$ & $1 / 1280$ & - & - \\
\hline 14 & 130 & 90 & 105 & 28 & 106 & $1 / 80$ & $1 / 1280$ & - & - \\
\hline 15 & 100 & 90 & 80 & 19 & 99 & $1 / 160$ & $1 / 1280$ & B.m. & B.m. \\
\hline 16 & 150 & 70 & 108 & 26 & 89 & $1 / 80$ & $1 / 640$ & - & - \\
\hline 17 & 240 & 90 & 98 & 36 & 88 & $1 / 160$ & $1 / 1280$ & - & - \\
\hline 18 & 180 & 100 & 103 & 27 & 109 & $1 / 80$ & $1 / 640$ & - & - \\
\hline 19 & 240 & 140 & 106 & 32 & 98 & $1 / 80$ & $1 / 640$ & - & - \\
\hline 20 & 170 & 110 & 180 & 32 & 94 & $1 / 80$ & $1 / 320$ & - & - \\
\hline 21 & 80 & 60 & 98 & 43 & 139 & $1 / 40$ & $1 / 640$ & - & - \\
\hline 22 & 150 & 90 & 101 & 23 & 89 & $1 / 320$ & $1 / 640$ & - & - \\
\hline 23 & 220 & 140 & 129 & 21 & 103 & $1 / 320$ & $1 / 1280$ & B.m. & B.m. \\
\hline 24 & 170 & 80 & 130 & 30 & 95 & $1 / 160$ & $1 / 1280$ & - & - \\
\hline 25 & 210 & 110 & 80 & 46 & 64 & $1 / 80$ & $1 / 1280$ & B.m. & B.m. \\
\hline
\end{tabular}

In our study, polyradiculopathy was determined in two patients who had sphincter dysfunctions and sensory loss in extremities resulting in walking difficulty. EMG was performed in these patients and sensory motor polyradiculopathy in the lower extremities as a result of axonal degeneration was detected. These two patients were discharged with sequel after being treated for six months with ceftriaxone plus rifampicin plus doxycycline.

In our patients, four different regimens were used. While ceftriaxone plus rifampicin plus cotrimaxosole was given to 11 (44\%) patients, ceftriaxone plus doxycycline plus cotrimaxosole was given to 8 (32\%) patients. Additionally, doxycycline plus rifampicin plus streptomycin and rifampicin plus streptomycin plus trimethoprim were given to $5(20 \%)$ and 1 (4\%) patient, respectively. Ceftriaxone was given in an intravenous dose of $100 \mathrm{mg} / \mathrm{kg} /$ day, rifampicin in an oral dose of $20-40 \mathrm{mg} / \mathrm{kg} /$ day, maximum $600 \mathrm{mg} /$ day, cotrimaxosole in an oral dose of $15 \mathrm{mg}$ / $\mathrm{kg} /$ day, doxycycline in an oral dose of $5 \mathrm{mg} / \mathrm{kg} /$ day in 2 divided doses and streptomycin in an intramuscular dose of $20 \mathrm{mg} /$ $\mathrm{kg}$ /day once daily. In these treatment regimens, ceftriaxone and streptomycin were given for one month. The remaining agents in these regimens were given together for a minimum of three months and maximum of sixth months while the mean duration time was $4.1 \pm 0.9$ months.

The mean follow-up duration was $29.8 \pm 17.4$ months with a range of 3 months to 60 months. One ( $4 \%$ ) patient who was given ceftriaxone plus rifampicin plus trimethoprim, died on the fifth day of hospitalization. Three patients had sequel. One of them, namely a seven-year-old girl with hydrocephalus and infarction had a ventriculoperitoneal shunt inserted in the third month of follow up because of hydrocephalus. Other two boys aged fourteen and fifteen with polyradiculopathy could hardly walk when unassisted. Their follow up after treatment took six and eight months, respectively. They failed to appear for control thereafter. The remaining 21 (84 $\%)$ patients were discharged with no sequel.

\section{Discussion}

Brucellosis remains an important public health problem in Turkey and it can affect people at any age, including children who represent $20-25 \%$ of cases $(3,13)$. Being endemic in Turkey, $B$. melitensis is isolated in most of children with brucellosis (14). In our study, one type of $B$. melitensis, was isolated from blood and cerebrospinal fluid cultures and $20 \%$ of patients were found culture-positive. These data were in compliance with literature. Epidemiological analysis of cases with brucellosis showed that living in rural area, consumption of unpasteurized dairy products or contact with infected animals or their waste materials are the main routes of infection (14-17). In our study, most of the patients had an epidemiological risk factor for brucellosis. Ninety-two per cent of all patients were from rural areas, $60 \%$ had been consuming unpasteurized dairy products, $56 \%$ had a history of brucellosis in their family members and $68 \%$ of patients had members of their 
family working either as farmers or herdsmen. These data were also consisted with literature.

In a study which was performed by Salari et al, it was found that brucellosis was most prevalent in summer (39.5\%) and more common in males than in females (16). Another study found that direct contact with animals, season, and gender represented the risk factors (17). A majority of cases were diagnosed between December and June (17). In other studies from Europe, $>70 \%$ of cases with brucellosis occurred from March to June with the peak observed from May to June $(18,19)$. All of these studies suggested that this seasonal variation could be related to the period when animals give birth to their young. During the time of deliveries, the numbers of animals excreting the infectious agents increase and the environmental contamination builds up progressively (17). In present study, male predominance was found $(\mathrm{p}<0.001)$ and the distribution of cases showed density in June (20\%) and February (20\%). These data were also consistent with literature. On the other hand, no data on seasonal variation in neurobrucellosis have been published. Nevertheless, we think that neurobrucellosis seasonal variations are associated with brucellosis seasonal variation. In adults, the male predominance is associated with occupational status (20). In older children, the probable explanation is that older boys are more involved in animal care (20). In our study however, we observed most of the cases to occur predominantly in boys between the age of 5 to 9 years and we were unable to explain why the latter age group was affected the most.

Neurobrucellosis is an uncommon complication of brucellosis (4-11\%) (5-7). It can present as meningitis, encephalitis, polyradiculopathy, brain abscess, epidural abscess, meningovascular complications, peripheral neuropathy, and psychosis $(21,22)$. The most common presentation of neurobrucellosis is acute meningitis (23). In our series, meningitis was observed in 23 (92\%) patients and polyradiculopathy in two (8\%) patients. Acute brucella meningitis is usually characterized by sudden onset of fever, headache, and nuchal rigidity (13). Psychiatric and motor-sensory disorders are also common (13). In our patients, fever, headache, sweating, and weight loss represented the majority of complaints, followed by neurological symptoms. On physical examination, meningeal irritation signs were seen in all patients. Other neurological abnormalities as confusion, hypoesthesia, convulsions, diplopia, papilledema, coma, and ataxia were seen in varying ratios. Being frequently seen in systemic brucellosis, hepatomegaly and splenomegaly, in our series were found to represent $20 \%$ and $16 \%$, respectively. We found that hepatomegaly and splenomegaly were uncommon in cases of neurobrucellosis. These data were consistent with literature. Similar findings were also reported by Gul HC et al (24). On the other hand, similar symptoms and findings may present in some patients with systemic brucellosis without neurologic involvement, and in those suffering from infectious meningitis due to other microorganisms (13). Therefore the diagnosis of neurobrucellosis requires demonstration of meningeal inflammation, abnormal CSF findings, and direct or indirect evidence of Brucella spp. in CSF $(11,12,33)$. In our study, these criteria were considered in the diagnosis of neurobrucellosis. Abnormal CSF findings were found in all patients. Pleocytosis was found in $96 \%$ of all patients. Although all patients have positive CSF tube agglutination test, $20 \%$ of patients had positive CSF and blood cultures for $B$. melitensis like Gul $\mathrm{HC}$ et al study in which CSF and positive blood cultures were found in $14 \%$ and $28 \%$ of patients, respectively (24). In Gul et al study, tube agglutination test was found to be positive in $81 \%$ in blood and $71 \%$ in CSF. In our study, we also observed that although all patients had abnormal CSF findings, $20 \%$ of patients had normal CSF glucose levels. Haji-Abdolbagi et al reported similar laboratory findings (13). In their study, all patients with meningitis had pleocytosis in CSF and in the majority of these patients not all; CSF protein and glucose were increased and decreased, respectively. These data showed that CSF glucose might be normal in neurobrucellosis and that this cannot serve as an exclusion criterium in the diagnosis of neurobrucellosis. Nevertheless, all patients had abnormal CSF findings especially with pleocytosis and positive CSF tube agglutination test. Therefore, our findings show that negative CSF tube agglutination test and negative CSF and blood culture may be used to exclude neurobrucellosis.

In our study, we observed that $24 \%$ of patients suffered from diplopia and sixth cranial nerve involvement. Cranial nerve involvement was reported in $19 \%$ of patients by Gul et al (24). They also found that the most affected nerves included the sixth and eighth cranial nerves (24). Abducens palsy occurs due to lateral rectus muscle paresis and eventuates in horizontal diplopia. This nerve is susceptible to direct and indirect insults as microvascular infarction or direct compression because of its longest intracranial course (25). However, cranial nerve palsies due to neurobrucellosis usually resolve completely with treatment $(8,26,27)$. Also in our study, all patients with cranial nerve involvement completely recovered after treatment. In our study, blurred vision was found in $16 \%$ of cases. In neurobrusellosis, there are two reasons of blurred vision, namely papilledema and ischemic vasculitis affecting the vasa nervorum of the optic nerve (28-30). Papilledema was determined in two ( $8 \%$ ) patients; in the remaining patients the blurred vision might have occurred due to ischemic vasculitis. In our study, the ratios of papilledema and blurred vision was found to be higher than those mentioned by Gul et al (24). The limited participation can be the reason of these high rates.

In our study, hydrocephalus with infarction, and brain abscess were observed in two patients. Cerebrovascular disease in neurobrucellosis has already been recorded in literature (12). Hemorrhages, transient ischemic attacks, and venous thrombosis are meningovascular complications of neurobrucellosis $(31,32)$. As for concurrent finding of hydrocephalus and infarction in association with neurobrucellosis, no cases have been reported in literature so far, while hydrocephalus alone has already been, albeit seldom, reported (33-35). In addition, neurobrucellosis presenting with a focal brain mass has been seldom demonstrated by imaging studies (36). These two rare complications were seen in our patients; one of these patients suffering from hydocephalus and infarction was discharged with sequel. In our study, polyradiculopathy was determined in two patients who had sphincter dysfunctions and sensory loss in extremities. Other two patients were admitted to our hospital due to memory deficit. Disturbances 
of orientation, memory and attention, as well as poverty of content can be seen in neurobrucellosis (37). Myelitis, which is generally evidenced by back pain, ataxia, paresthesia, paraplegia, and sphincter abnormalities, is seen in neurobrucellosis with a ratio lower than $5 \%(38)$.

The treatment of neurobrucellosis included concurrent administration of three of the drugs as follows: doxycycline, rifampicin, streptomycin, cotrimaxosole, ceftriaxone, or ciprofloxacin (8). Doxycycline ( $5 \mathrm{mg} / \mathrm{kg} /$ day in 2 divided peroral doses) with intramuscular streptomycin $(20 \mathrm{mg} / \mathrm{kg} /$ day once daily) or gentamicin $(3-5 \mathrm{mg} / \mathrm{kg}$ /day in 2 divided slow intravenous or intramuscular doses) with peroral rifampicin (600-900mg/day) and/or prednisolone $(20 \mathrm{mg} /$ day in divided peroral doses) are also recommended (39). In our study four different regimens were used: (a) ceftriaxone plus rifampicin plus cotrimaxosole, (b) ceftriaxone plus doxycycline plus cotrimaxosole, (c) doxycycline plus rifampicin plus streptomycin, and (d) rifampicin plus streptomycin plus cotrimaxosole. All of these used regimens were found effective.

\section{Conclusion}

In conclusion, we showed that clinical manifestations of neurobrucellosis vary widely while meningitis is the most frequent manifestation. We would like to emphasize the fact that clinicians, especially those providing health services in endemic areas as Turkey, should keep neurobrucellosis in mind when treating patients with unexplained symptoms such as memory impairment, or patients diagnosed with meningitis. In all such cases they should perform the necessary tests on blood and CSF. People living in areas endemic for brucellosis should be kept informed about decontamination and eradication of Brucella.spp as well as on the necessity to cease consuming unpasteurized dairy products.

\section{What already is known}

1. Hepatomegaly and splenomegaly are generally found in brucellosis.

2. In neurobrucellosis, lymphocyte supremacy is found in cerebrospinal fluid examination.

3. Hydrocephalus with infarction in neurobrucellosis has not been reported in literature, while hydrocephalus in neurobrucellosis has seldom been reported.

\section{What this paper adds}

1. Hepatomegaly and splenomegaly are rarely found in association with neurobrucellosis

2. In neurobrusellosis, polymorphonuclear supremacy is sometimes found in CSF examination. Therefore, clinicians, especially those giving health services in endemic areas like Turkey, should keep neurobrucellosis in mind when treating patients with purulent meningitis, and should thus perform the necessary blood and CSF tests.

3. A case of hydrocephalus with infarction in association with neurobrucellosis was found in present study; therefore patients with hydrocephalus and/or infarction should be evaluated in terms of neurobrucellosis.

\section{References}

1. Skalsky K, Yahav D, Bishara J, Pitlik S, Leibovici L, Paul M. Treatment of human brucellosis: systematic review and meta-analysis of randomised controlled trials. BMJ 2008; 336: 701-704.

2. Centers for Disease Control and Prevention. Available at: http://www. cdc.gov/ncidod/dbmd/diseaseinfo/brucellosis_g.htm\#-howcommon. (accessed April 2009).

3. Ministry of Health of Turkey. General Directorate of Primary Health Services. Available at: http://www.saglik.gov.tr (accessed 01.12.2005).

4. Mantur BG, Mangalgi SS, Mulimani M. Brucella melitensis--a sexually transmissible agent? Lancet 1996; 347: 1763.

5. Yüce A, Alp-Çavuslu S, Yapar N, Çakır N. Brucellosis: Evaluation of 55 case. Klimik Derg 2006; 19: 13-17.

6. Cesur S, Capar Y, Demir P et al. A retrospective evaluation of 104 cases of brucellosis. Turk J Infect 2000; 18: 169-173.

7. Geyik MF, Kökoğlu ÖF, Hosoglu S, Ayaz C. The evaluation of 154 patients with brucellosis. Dicle Med J 2002; 29: 23-29.

8. Akdeniz H, Irmak H, Anlar O, Demiröz AP. Central nervous system brucellosis: presentation, diagnosis and treatment. J Infect 1998; 36: 297301.

9. Gotuzzo E, Carrillo C. Brucella. 1717-1742. In: Gorbach SL, Bartlett JG, Blacklow NR (Eds). Infectious diseases. 3rd ed. Philadelphia: Lippincott, Williams \& Wilkins; 2004.

10. Shakir RA, Al-Din AS, Araj GF, Lulu AR, Mousa AR, Saadah MA. Clinical categories of neurobrucellosis. A report on 19 cases. Brain. 1987 ; 110: 213-223.

11. McLean DR, Russell N, Khan MY. Neurobrucellosis: clinical and therapeutic features. Clin Infect Dis 1992; 15: 582-590.

12. Bouza E, García de la Torre M, Parras F, Guerrero A, RodríguezCréixems M, Gobernado J. Brucellar meningitis. Rev Infect Dis 1987; 9: 810-822.

13. Haji-Abdolbagi M, Rasooli-Nejad M, Jafari S, Hasibi M, Soudbakhsh A. Clinical and laboratory findings in neurobrucellosis: review of 31 cases. Arch Iran Med 2008; 11: 21-25.

14. Mantur BG, Akki AS, Mangalgi SS, Patil SV, Gobbur RH, Peerapur BV. Childhood brucellosis - a microbiological, epidemiological and clinical study. J Trop Pediatr 2004; 50: 153-157.

15. Shaalan MA, Memish ZA, Mahmoud SA et al. Brucellosis in children: clinical observations in 115 cases. Int J Infect Dis 2002; 6: 182-186.

16. Salari MH, Khalili MB, Hassanpour GR. Selected epidemiological features of human brucellosis in Yazd, Islamic Republic of Iran: 19931998. East Mediterr Health J 2003; 9: 1054-1060

17. Minas M, Minas A, Gourgulianis K, Stournara A. Epidemiological and clinical aspects of human brucellosis in Central Greece. Jpn J Infect Dis 2007; 60: 362-366

18. Kolar J (1989). Brucellosis in Eastern European Countries. P.163-172. In Young, E., Carbel , M. (eds) Brucellosis: Clinical and Laboratory Aspects CRC Press, Boca Raton, Florida.

19. Abela B. Epidemiology and control of brucellosis in ruminants from 1986 to 1996 in Malta. Rev Sci Tech 1999; 18: 648-659.

20. Tanir G, Tufekci SB, Tuygun N. Presentation, complications, and treatment outcome of brucellosis in Turkish children. Pediatr Int 2009; 51: $114-9$ 
424-430

21. Yilmaz M, Ozaras R, Ozturk R, Mert A, Tabak F, Aktuglu Y. Epileptic seizure: an atypical presentation in an adolescent boy with neurobrucellosis. Scand J Infect Dis 2002; 34: 623-625.

22. Haji-Abdolbagi M, Rasoolinejad M, Yaghoubzadeh M, Louti Shahrokh B. Epidemiological, clinical, diagnostic, and therapeutic survey in 505 cases with brucellosis. J Tehran Facul Med 2001; 4: 34-46.

23. Shakir RA. Neurobrucellosis. Postgrad Med J 1986; 62: 1077-1079

24. Gul HC, Erdem H, Bek S. Overview of neurobrucellosis: a pooled analysis of 187 cases. Int J Infect Dis 2009 May 8.

25. Danchaivijitr C, Kennard C. Diplopia and eye movement disorders. J Neurol Neurosurg Psychiatry 2004; 75: 24-31.

26. Gouider R, Samet $\mathbf{S}$, Triki $\mathbf{C}$ et al. Neurological manifestations indicative of brucellosis. Rev Neurol (Paris) 1999; 155: 215-218.

27. Pascual J, Combarros O, Polo JM, Berciano J. Localized CNS brucellosis: report of 7 cases. Acta Neurol Scand 1988; 78: 282-289.

28. Yilmaz M, Ozaras R, Mert A, Ozturk R, Tabak F. Abducent nerve palsy during treatment of brucellosis. Clin Neurol Neurosurg 2003; 105: 218-220.

29. Miyares FR, Deleu D, EIShafie SS et al. Irreversible papillitis and ophthalmoparesis as a presenting manifestation of neurobrucellosis. Clin Neurol Neurosurg 2007; 109: 439-441

30. al Deeb SM, Yaqub BA, Sharif HS, Phadke JG. Neurobrucellosis: clinical characteristics, diagnosis, and outcome. Neurology 1989; 39 : 498-501.

31. Bingöl A, Togay-Ișikay C. Neurobrucellosis as an exceptional cause of transient ischemic attacks. Eur J Neurol 2006; 13: 544-548.
32. Yetkin MA, Bulut C, Erdinc FS, Oral B, Tulek N. Evaluation of the clinical presentations in neurobrucellosis. Int J Infect Dis 2006; 10: 446-452.

33. Guney F, Gumus H, Ogmegul A et al. First case report of neurobrucellosis associated with hydrocephalus. Clin Neurol Neurosurg 2008; 110: 739-742.

34. Diaz Espejo CE, Villalobos Chaves F, Sureda Ramis B. Chronic intracranial hypertension secondary to neurobrucellosis. J Neurol 1987; 234: 59-61.

35. Anderson H, Mortensen A. Unrecognised neurobrucellosis giving rise to Brucella melitensis peritonitis via a ventriculoperitoneal shunt. Eur J Clin Microbiol Infect Dis 1992; 11: 953-954.

36. Martínez-Chamorro E, Muñoz A, Esparza J, Muñoz MJ, Giangaspro E. Focal cerebral involvement by neurobrucellosis: pathological and MRI findings. Eur J Radiol 2002; 43: 28-30.

37. Eren S, Bayam G, Ergönül O et al. Cognitive and emotional changes in neurobrucellosis. J Infect 2006; 53: 184-189.

38. Krishnan C, Kaplin AI, Graber JS, Darman JS, Kerr DA. Recurrent transverse myelitis following neurobrucellosis: immunologic features and beneficial response to immunosuppression. J Neurovirol 2005; 11: $225-231$.

39. Samdani PG, Patil S Neurobrucellosis. Indian Pediatr 2003; 40: $565-568$.

Received January 10, 2010. Accepted April 15, 2012. 